American Journal of Applied Sciences 6 (12): 2066-2070, 2009

ISSN 1546-9239

(C) 2009 Science Publications

\title{
A Novel Morphological Method for Detection and Recognition of Vehicle License Plates
}

\author{
${ }^{1}$ S.H. Mohades Kasaei, ${ }^{1}$ S.M. Mohades Kasaei and ${ }^{2}$ S.A. Monadjemi \\ ${ }^{1}$ Young Researchers Club, Department of Computer, Islamic Azad University, \\ Khourasgan Branch, Isfahan, Iran \\ ${ }^{2}$ Department of Computer Engineering, Faculty of Engineering, University of Isfahan, \\ Isfahan, 81746, Iran
}

\begin{abstract}
Problem statement: License plate detection and recognition is an image-processing technique used to identify a vehicle by its license plate. This notable technology has got multiple applications in various traffic and security cases. To name but a few, toll roads, border control, security and car tracking are same of its applications. The main stage is the isolation of the license plate, from the digital image of the car obtained by a digital camera under different circumstances such as illumination, slop, distance and angle. Approach: This study presented a novel method of identifying and recognizing license plates based on the morphology and template matching. The algorithm started with preprocessing and signal conditioning. Next license plate is localized using morphological operators. Then a template matching scheme will be used to recognize the digits and characters within the plate. Results: The system was tested on Iranian car plate images and the performance was $97.3 \%$ of correct plates identification and localization and $92 \%$ of correct recognized characters. The results regarding the complexity of the problem and diversity of the test cases showed the high accuracy and robustness of the proposed method. The method could also be applicable for other applications in the transport information systems, where automatic recognition of registration plates, shields, signs and so on is often necessary. Conclusion: This system was customized for the identification of Iranian license plates. The results showed that this algorithm performs well on different types of vehicles including Iranian car and motorcycle plates as well as diverse circumstances. We believe that this system can be redesigned and tested for multi national car license plates in the future time regarding their own attributes.
\end{abstract}

Key words: License plate recognition, character recognition, object localization, transport information systems, segmentation

\section{INTRODUCTION}

While the first industrial automatic system for Car License Plate Recognition (LPR) was introduced in the 80's, an outburst of commercial systems occurred in the 90s. Although that a lot of LPR systems are available in the market, the research and development still continues and new sophisticated solutions to plate localization, character segmentation and recognition appear.

This is due to the growing demand for the automatic vehicle identification required for traffic control, border control, access-control, calculation of parking time and payment, search for stolen cars or unpaid fees and the requirement for reliable identification at different lighting conditions, presence of random or structured noise in the plate and nationality specific features, concerning plate's size and type of characters ${ }^{[1,2]}$. Figure 1 shows a typical license plate recognition system.

Some of the related research in the field of automatic car plate recognition are as in Fig. 1.

Lotufo, Morgan and Johnson ${ }^{[2]}$ proposed automatic number-plate recognition using optical character recognition techniques. Abolghasemi and Ahmadifard ${ }^{[5]}$ proposed an IFT-based fast method for extracting the license plate. Meanwhile Zamani and Movahedi ${ }^{[6]}$ proposed a morphologic method for extracting the license plate using some simple constant templates.

Corresponding Author: S.H. Mohades Kasaei, Young Researchers Club, Department of Computer, Islamic Azad University, Khourasgan Branch, Isfahan, Iran Tel: +98 3117934035 Fax: +98 3117932670 
Am. J. Applied Sci., 6 (12): 2066-2070, 2009

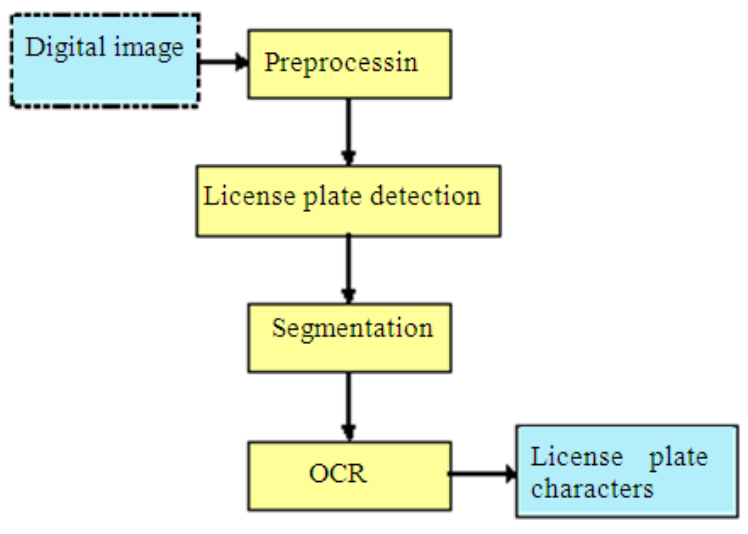

Fig. 1: An automatic vehicle License Plate Recognition system

In this study, we introduce a novel method for license plate recognition from a grayscale image of the car. We first use a preprocessing stage that contains a certain number of transforms established heuristically for reduces the extra information of car picture. Input of the car plate detection and recognition system is the binary image that obtains form preprocessing stage. Next, start a process for identifying and isolation of the car's license plate from the image, then the result of this process send to segmentation part. Segmentation part separates the characters individually. Finally recognition part recognizes the characters giving the result as the plate number.

\section{MATERIALS AND METHODS}

Based on their technical knowledge, expertise, level of potency and available means educational institutes and organizations, have several solutions to implement an E-learning system. In the following sections, we will pay special attention to these solutions which are one of two kinds: Purchasing a software program or designing one.

Preprocessing: Preprocessing mostly is necessary to facilitate further high performance recognition. In this study, we firstly convert the RGB color input image to a 256 grayscale image using Eq. 1:

$\mathrm{A}_{\mathrm{GL}}=\frac{3 \mathrm{~A}_{\mathrm{R}}+6 \mathrm{~A}_{\mathrm{G}}+\mathrm{A}_{\mathrm{B}}}{10}$

Where:

$\mathrm{A}_{\mathrm{GL}} \quad=$ The converted gray level image

$A_{R}, A_{G}$ and $A_{B}=R, G$ and $B$ spectrum of the color image respectively
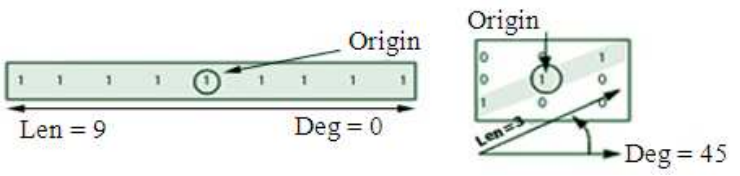

Fig. 2: Structural elements for horizontal and $\theta=45$ rotated line

Next, a vertical and horizontal Sobel operator is applied on the image to amplify the corresponding edges. Then an appropriate threshold $\mathrm{T}$ is used to generate the binary images where the edges are highlighted in a black background. (Eq. 2):

$G(x, y)=\left\{\begin{array}{lr}1 & \text { ifA }(x, y) \geq T \\ 0 & \text { other_wise }\end{array}\right.$

The experiments suggest that the threshold $\mathrm{T}$ must be from nearly 0.25 for clear, noise free, bright environments to almost 0.12 for noisy, darker environments. The mean value of the main image is used to determine whether it is bright enough or not, while the difference between the energies of the image and its one octave low pass filtered version determines the level of noise. A dilation operator also is used to generate more continues borders and edges. Equation 3 illustrates the dilation operation, where A and B are the image and the structuring element respectively:

$$
\mathrm{A} \oplus \mathrm{B}=\left\{\mathrm{d} \in \mathrm{R}^{2}: \mathrm{d}=\mathrm{a}+\mathrm{b}, \forall \mathrm{a} \in \mathrm{A}, \mathrm{b} \in \mathrm{B}\right\}
$$

Plate region extraction: After the preprocessing stage, a morphological operator is applied to the image for specifying the plate location. Morphology is a technique in image processing based on shapes. We can build a morphological operator that be sensitive to a specific shape in the input image. In fact we try to create a structural element with special properties. For instance, a horizontal line of length $=5$ pixels or a rotated one with $\theta=45$ can be some typical structural elements. In this study, a rectangular box can be employed as a structural element to detect the car plates (Fig. 2).

Applying the closing operation on edge image in the horizontal direction yields several connected regions which are plate candidates. With respect to a license plate shape, a rectangular Structural Element (SE) is used. The main motivation to focus on morphology was the rectangle shape of the plates. However, there may be more than one candidate region as plate location in the image after closing. 


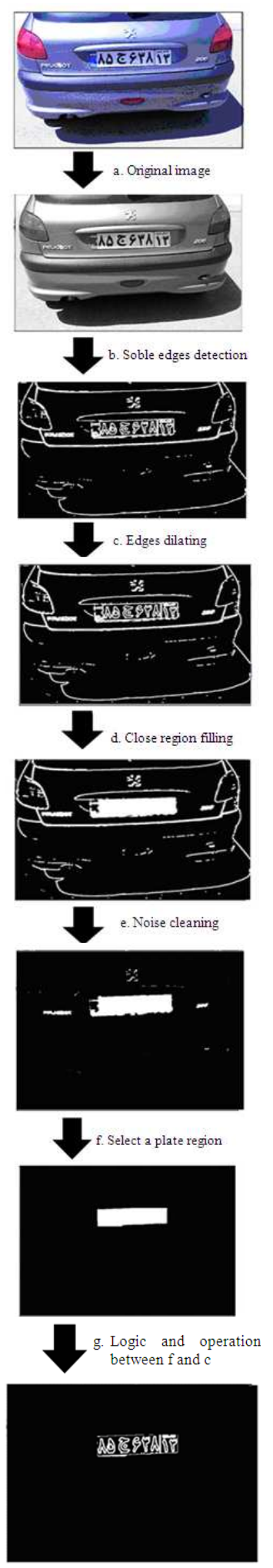

Fig. 3: Vehicle plate detection procedure

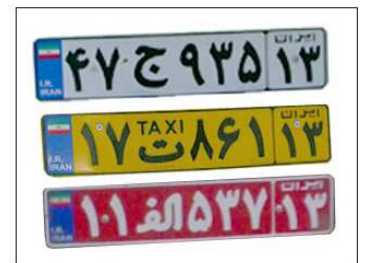

Fig. 4: Some samples of Iranian car plates

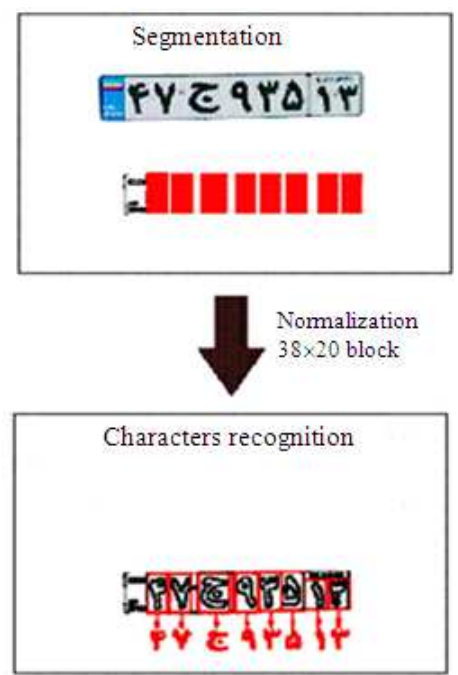

Fig. 5: Segmentation, normalization and the final character recognition

To find the correct region and discard the others, some features such as shape, aspect ratio and size of the plate are tested for all regions; this filter considerably reduces the Noise. The processed image after these stages is as shown in Fig. 3, while Fig. 4 illustrates some Iranian care plates.

Segmentation: Character segmentation is an important stage in many license plate recognition systems. There are many factors that cause the character segmentation task difficult, such as image noise, plate frame, rivet and rotation and illumination variance ${ }^{[4,5]}$. Preprocessing is very important for the good performance of character segmentation. Firstly, image is filtered for enhancing the image and removing the noises and unwanted spots. Then dilation operator is applied to the image for separating the characters from each other if the characters are close. After this stage, partition scanning is proposed to extract characters from plate. This method is conducted by checking the mean of each partition in image (the size of partition of a character or background. In this process, the 
background is defined as white (1) and characters are defined as black (0). After some experiments, it is concluded that the optimum threshold value is nearly $0.7-0.8$. Partition value larger than 0.7-0.8 is considered as background, otherwise it is considered as character. Then the plate is divided into two blocks. The first block contains digits and the second block contains the letter (Fig. 4).

Character recognition using template matching: Before employment of the recognition algorithm, the characters would be normalized. Normalization is to refine the characters into a block containing no extra white spaces (pixels) in all the borders of the characters. Then each character is fit to a $38 \times 20$ block as shown in Fig. 5.

Fitting approach is also necessary for template matching. For matching the characters with the database, input images must be equalizing to a $38 \times 20$ block with the database characters. In this system the characters are fit to $38 \times 20$. The extracted characters cut from plate and the characters on database are now equalized. The next step is template matching. Template matching is an effective algorithm for recognition of characters. The characters' image is compared to the ones in the database and the best similarity is considered. To measure the similarity and find the best match, a statistical method correlation based is used. Correlation is an effective technique for image recognition. This method measures the correlation coefficient between a number of known images with the same size unknown images or parts of an image with the highest correlation coefficient between the images producing the best match. This system used the database as the Iranian license plates characters all 25 alphanumeric characters (16 alphabets and 9 numerals) with the size of $38 \times 20$. Due to the similarities of some characters, there may be some errors during the recognition phase. The confused characters mainly are "ت" and To increase the recognition rate, some properties of each character are used in the system for the confused one to define their special feature of vectors. With these feature the applied tests show a serious increase in the correct recognition rate.

\section{RESULTS}

Experiments have been performed to test the proposed system and to measure the accuracy of the system. The input images are colored images with the size of $640 \times 480$. The test images were taken under various illumination conditions and distance. The results of the tests are presented in Table 1.
Table 1: Experiments results

\begin{tabular}{lll}
\hline Applied stages & No. of correct detection & Accuracy (\%) \\
\hline Extraction of plate region & $146 / 150$ & 97.3 \\
Segmentation & $141 / 150$ & 94.0 \\
Recognition of characters & $138 / 150$ & 92.0 \\
\hline
\end{tabular}

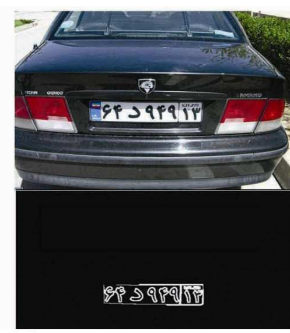

sks qkq I

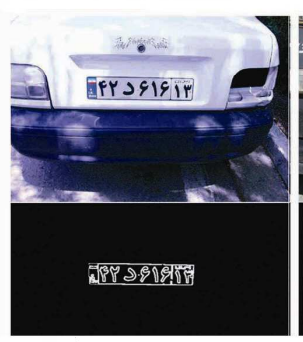

ERS GIS IN

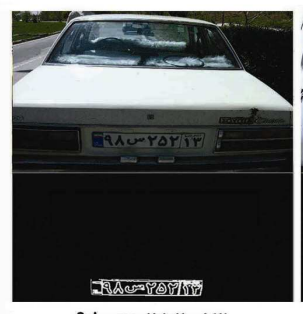

१^

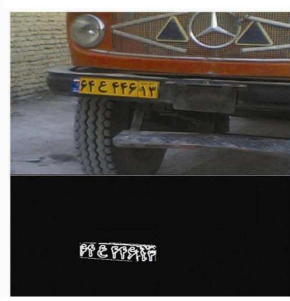

SF E KFS I

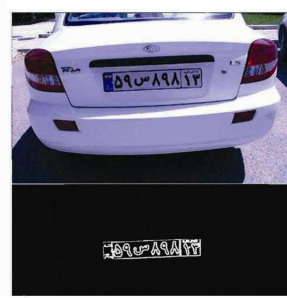

ด१ $\wedge$ ผ ।

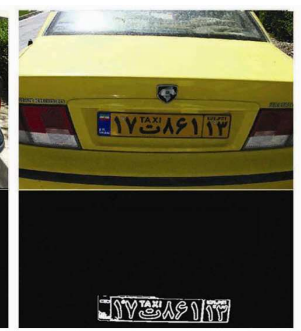

$|V N \wedge S|$ I

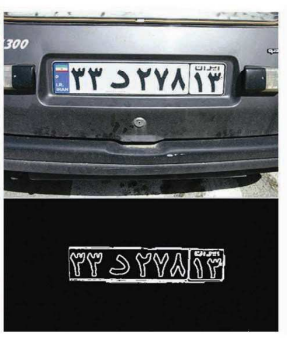

د

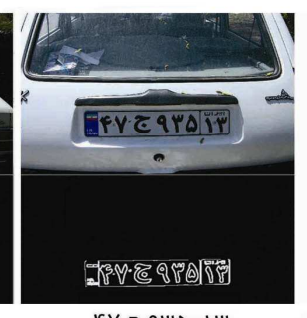

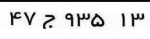

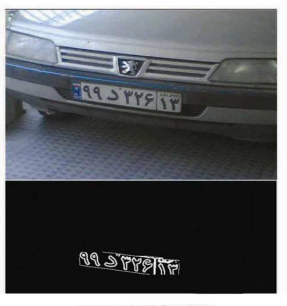

११> HसS I

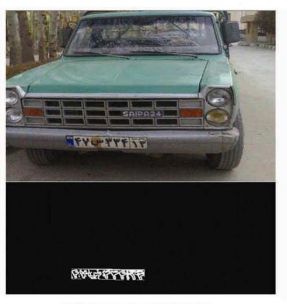

cV w r p i
Fig. 6: The outcomes of the LPR system, car images, localized car plate and recognized characters in different test images 
It is shown that accuracy for the extraction of plate region is 97.3 and $94 \%$ for the segmentation of the characters and $92 \%$ is the percentage of accuracy of the recognition unit. Tested on ten different cars, Fig. 6 shows the outcomes of the system.

\section{DISCUSSION}

A new method for car license plate detection and recognition system are presented in this study, generally accenting on the problems of License Plate Localization (LPL) instead of the LPCR (License Plate Character Recognition) therein. This reflects the License Plate Localization (LPL) specifics of CLPR application, where the problems of LPCR are usually considered priori resolved by usage of conventional Optical Character Recognition (OCR) software. The goal of the research is to investigate the possibility to create a comprehensive system for multinational vehicle identification based on the license plate recognition. In that case no additional hardware such as transmitters mounted on the vehicle or additional sensors are required. The preliminary results obtained on real data are quite satisfactory.

\section{CONCLUSION}

In this study, we presented a novel method of identifying and recognizing of Iranian car license plates. Firstly we extracted the plate location and then we separated the plate characters by segmentation and applied a correlation based template matching scheme for recognition of plate characters. This system is customized for the identification of Iranian license plates. The system is tested over a large number (more than 150) of images, where this algorithm performs well on different types of vehicles including Iranian car and motorcycle plates as well as diverse circumstances. Finally it is proved to be $97.3 \%$ correct in the extraction of plate region and $94 \%$ correct in the segmentation of the characters and $92 \%$ in the recognition of the characters. We believe that this system can be redesigned and tested for multi national car license plates in the future time regarding their own attributes.

\section{REFERENCES}

1. Ozbay, S. and E. Ercelebi, 2005. Automatic vehicle identification by plate recognition. World Acad. Sci. Eng. Technol., 9: 222-225. DOI: 10.1177/016224399201700404

2. Lotufo, R.A., A.D. Morgan and A.S. Johnson, 1990. Automatic number-plate recognition. Proceedings of the IEEE Colloquium on Image Analysis for Transport Applications, Feb. 16-16, IEEE Xplore Press, London, UK., pp: 1-6. http://ieeexplore.ieee.org/xpl/freeabs_all.jsp?arnum ber $=191011$

3. Bailey, D.G., D. Irecki, B.K. Lim and L. Yang, 2002. Test bed for number plate recognition applications. Proceedings of the 1st IEEE International Workshop on Electronic Design, Test and Applications, Jan. 29-31 IEEE Computer Society, Christchurch, New Zealand, pp: 501-503. DOI: 10.1109/DELTA.2002.994684

4. Jain, A. and B. Yu, 1998. Automatic text location in images and video frames. Proceedings of the 14th International Conference on Pattern Recognition, Aug. 16-26, IEEE Xplore Press, Brisbane, Qld., Australia, pp.1295-1297. DOI: 10.1109/ICPR.1998.711990

5. Abolghasemi, M. and A. Ahmadifard, 2007. A car plate identification system based on IFT transform. Proceedings of 3th Conference on Information and Knowledge Technology, Nov. 2007, Mashhad, Iran.

6. Zamani, A. and M. Movahedi, 2007. Car plate identification using morphology and constant moments transform. Proceedings of 3th Conference on Information and Knowledge Technology, Nov. 2007Mashhad, Iran. 